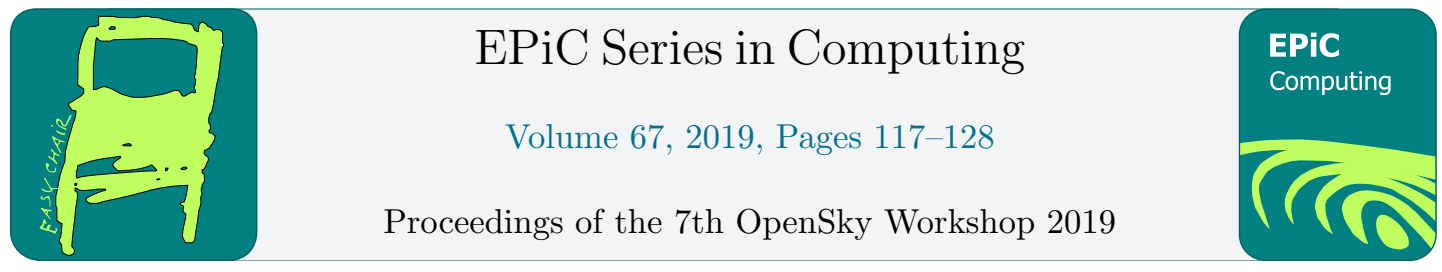

\title{
Higher than a Kite:ADS-B Communication Analysis Using a High-Altitude Balloon
}

\author{
Matthias Schäfer ${ }^{1}$, Roberto Calvo-Palomino ${ }^{2}$, Franco Minucci ${ }^{3}$, \\ Brecht Reynders ${ }^{3}$, Gérôme Bovet ${ }^{4}$, and Vincent Lenders ${ }^{45}$ \\ 1 SeRo Systems GmbH, Kaiserslautern, Germany - schaefer@sero-systems.de \\ 2 IMDEA Networks Institute, Madrid, Spain - roberto.calvo@imdea.org \\ 3 KU Leuven, Leuven, Belgium - $\{$ franco.minucci, brecht.reynders $\}$ @esat.kuleuven.be \\ 4 armasuisse, Switzerland - \{gerome.bovet, vincent.lenders\}@armasuisse.ch \\ 5 Electrosense, Switzerland
}

\begin{abstract}
Receiving signals on the $1090 \mathrm{MHz}$ frequency, one of the most important radio frequencies used in aviation, is typically done using ground-based receivers. However, an increasing number of airborne or even space-based receivers also aim to receive these signals for applications such as air traffic surveillance and collision avoidance. In this paper, we present our results from a high-altitude radio frequency measurement campaign with the goal to gain insights about the challenges and limitations of receiving $1090 \mathrm{MHz}$ signals at high altitudes. We used a high-altitude balloon equipped with a software-defined radio to collect $1090 \mathrm{MHz}$ signal data. In an extensive analysis of these data, we identify several challenges and provide a first impression of the radio environment at altitudes up to $33.5 \mathrm{~km}$.
\end{abstract}

\section{Introduction}

One of the arguably most important radio frequencies used in todays air transportation system is $1090 \mathrm{MHz}$. It provides the communication backbone for many crucial applications including air traffic surveillance and collision avoidance. Due to the frequency's high relevance and its unique characteristics and history, an increasing number of operational systems are relying on it, including military and civil Secondary Surveillance Radars (SSR Mode 1, 2, 3/A, C, S, 4, 5), Airborne Collision Avoidance Systems (ACAS), and the next generation surveillance technology Automatic Dependent Surveillance-Broadcast (ADS-B).

The main reason why this particular frequency is being reused even in newer systems is the strong need for legacy compatibility and cost efficiency. Unfortunately, the price for this legacy compatibility is the congestion and high interference levels experienced on $1090 \mathrm{MHz}$. Due to the excessive use of the frequency by a multitude of operational systems and the high number of transponders in the air, areas with high traffic densities are experiencing degradation of system performance or in extreme cases even system failures. One infamous example is the failure of several transponders across Europe in 2014 due to over-interrogation by ground radars [1]. 


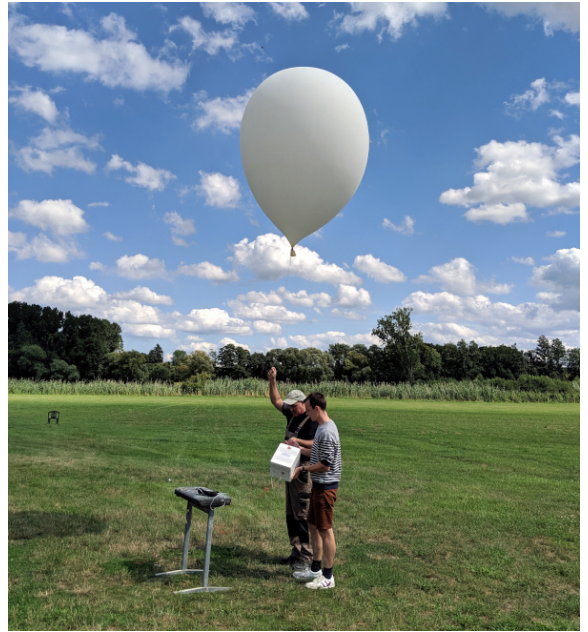

(a) High-altitude balloon used for the measurement campaign. The payload is put into a styrofoam enclosure for protection and insulation.

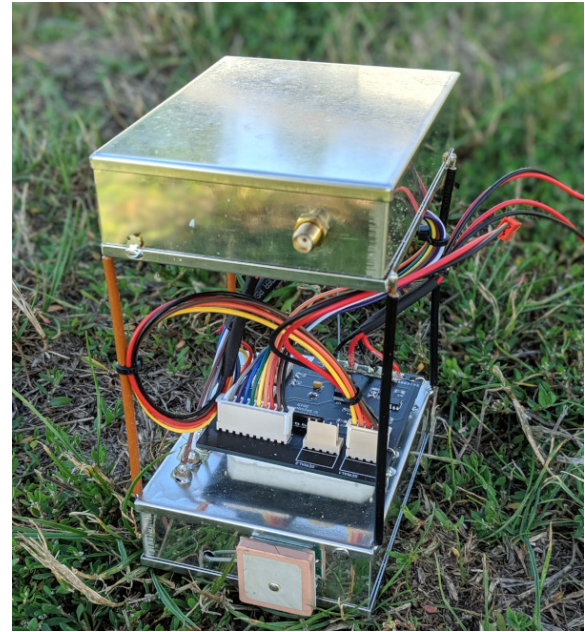

(b) The balloon's payload consisting of a tele-metry transmitter and controller system and a Raspberry Pi Zero attached to an RTL-SDR.

Figure 1: Setup for our high-altitude radio measurement campaign.

While the spectrum around $1090 \mathrm{MHz}$ on the ground is a well-studied field (e.g., [10]), little is publicly known about the usage of this frequency at higher altitudes. This is surprising given the increasing importance of $1090 \mathrm{MHz}$ signal reception at high altitudes that comes along with the ongoing development and deployment of space-based surveillance and more complex future versions of ACAS. However, we believe that the natural reason for this lack of information is that measurements at higher altitudes are simply not as easily accessible as those on the ground.

In this work we fill this gap by presenting the insights gained during a high-altitude $1090 \mathrm{MHz}$ frequency measurement campaign. More specifically, we used a high-altitude balloon equipped with a low-cost software-defined radio (SDR) to continuously record radio frequency data (I/Q samples) during the balloon's $33.5 \mathrm{~km}$ ascent. Based on the balloon's telemetry, we were able to recover the payload and the recorded data after it landed about $200 \mathrm{~km}$ from the launch site. By providing the results of our extensive post-mission data analysis, we aim at shedding light on the situation of the $1090 \mathrm{MHz}$ frequency at higher altitudes and the challenges associated with high-altitude spectrum measurements.

\subsection{Related Work}

In recent years, several attempts have been made for measuring ADS-B messages with HABs including $[4,3,2,6]$. These experiments all tried to hoover balloons on a high altitude close to 30 kilometer and measure ADS-B traffic at that altitude. The main goal was to see if satellites could cover the dead spots in ADS-B messaging system, such that safety could go up and fuel consumption could go down. The drawback of most of these studies is that they are done in places with few airplanes only. The authors mention up to 100 airplanes during the whole measurement. This does however result in huge coverage ranges, up to $850 \mathrm{~km}$. In our case, we investigate the region around Frankfurt airport with more avionic traffic.

Since the balloons hoovered on a high altitude, they ignored the altitudes below. The 
exception is [2], they did launch a balloon that measured the intermediate altitudes between the ground and their 30 kilometer altitude. The results are rather coarsely divided in one hour slots, so it is difficult to assess the effect of altitude with these results.

Interesting theoretical work has been done in $[12,7,8]$. All papers describe a theoretical framework to motivate the use of satellites to monitor ADS-B messages. In these they calculate the amount of missed ADS-B messages, the packet collision probability. Interesting results although some estimations are simplified heavily. It is important to note that lower orbit satellites, or high-altitude balloons have the advantage that they see more difference in the received signal strength. This will make the proposed simulations for balloons more challenging as the near-far problem needs to be taken into account. While in the simulations the power differences will be small and collisions are more likely.

Finally, it is remarkable to notice that the first launch of these papers usually fails, including ours. The reason for failure differ from paper to paper.

\section{Background}

In this section, we provide the relevant background on the frequency considered in this project and the systems using it. Moreover, we provide a quick introduction into the specific challenges of measurement campaigns with high-altitude balloons.

\section{$2.1 \quad 1090 \mathrm{MHz}$ Frequency}

Both ground-based secondary surveillance radars as well as airborne collision avoidance system are all sharing the same frequencies to localize aircraft within their operational range. Both systems transmit directed interrogations on $1030 \mathrm{MHz}$ which elicit the transmission of replies by airborne transponders on $1090 \mathrm{MHz}$. Since most aircraft are flying at high altitudes, reply transmissions often cover much wider areas than interrogations, leading to an increased frequency occupancy and interference level across large areas. To put this into perspective, surface interrogators usually have a rather narrow beam width of 2-4 degrees [11] whereas replies from aircraft can be received on the ground over distances of more than $400 \mathrm{~km}$ in all directions, depending on the aircraft's altitude and the surrounding geography.

These interferences caused by the long ranges and the multitude of high-altitude transmitters is amplified by the fact that older systems are rather inaccurate. As a consequence, interrogations are usually transmitted at high rates to obtain more measurements and sufficient update rates. Moreover, the older SSR Mode A/C and military Modes 1 and 2 do not use direct addressing in their interrogations, hence eliciting replies from many aircraft with each interrogation.

Besides SSR and ACAS, many transponders are additionally equipped with ADS-B. As ADS-B becomes mandatory for scheduled air traffic in 2020, about $90 \%$ of the transponders seen over Central Europe have been upgraded at the time of this writing. Once equipped with ADS-B, transponders elicit a specific type of SSR Mode S transmission without prior interrogation, adding additional load to the $1090 \mathrm{MHz}$ frequency. According to OpenSky ${ }^{1}$, about $20 \%$ of all Mode S transmissions observed by the network are caused by ADS-B.

Finally, in some regions, other sources of interferences on the $1090 \mathrm{MHz}$ exist such as the operation of Distance Measuring Equipment (DME) on nearby frequency bands.

\footnotetext{
${ }^{1}$ https://opensky-network.org/network/facts
} 


\subsection{High-altitude Balloons}

High-altitude balloons (HABs) offer a cheap way for high-altitude measurement campaigns compared to other common approaches such as measurement flights with aircraft. However, they come with some limitations which make experiments more challenging. In particular, HABs must have a low mass to stay within the regulatory limits that prevent them from causing damage in the unlikely event of a collision with air traffic or on impact on the ground. Moreover, each additional $100 \mathrm{~g}$ of weight result in about $1 \mathrm{~km}$ lower maximum altitude.

A consequence of this crucial low weight requirement is that the battery onboard the balloon's payload must be as small and light as possible, limiting the power supply significantly. Therefore, heavy high-end measurement equipment cannot be easily used due to the light weight and low power requirements.

\section{Setup \& Execution}

As previously mentioned, the size and weight of the HAB influences the attainable altitude. Thanks to the lightweight design and components used, the payload weights only $870 \mathrm{~g}$ with a dimension of 260x210x192 mm. The payload consists of Skysquitter's ${ }^{2}$ proprietary telemetry transmitter and control system and a Raspberry Pi Zero W connected to the well-known RTL$\mathrm{SDR}^{3}$ v3 software-defined radio receiver. We hereby rely on Electrosense [9] software running on the Raspberry Pi for signal acquisition through the RTL-SDR. All electronics were installed in a sheet metal enclosure to avoid any effect from cosmic radiation. The enclosure was then deployed in a styrofoam box for protection and insulation. Figure 1 shows the balloon along with the probe.

The RTL-SDR receiver was tuned to $1090 \mathrm{MHz}$ with a sample rate of $2.4 \mathrm{M}$ samples per second. The sample resolution was 8 bit. The antenna was a quarter wavelength copper litz wire. The Raspberry Pi onboard the HAB was configured to record the raw I/Q data provided by the RTL-SDR in $30 \mathrm{~s}$ intervals and store the data to an SD card. To allow the control system to transmit the transmissions of the balloon's telemetry and due to the limited power supply, the I/Q recordings where paused for about 1 minute after each $30 \mathrm{~s}$ recording interval.

\subsection{Flight Path}

The flight path of our radio probe is shown in Figure 2. It was recorded using the onboard GPS sensor. The balloon was launched on August 20, 2019 at 8:40am UTC from a small airfield about $30 \mathrm{~km}$ south-east from Frankfurt am Main Airport. It travelled $145 \mathrm{~km}$ north-east and, after almost 2 hours ascending, it burst at an altitude of 33,565 m (10:33am UTC). After another 32 minutes descent, at 11:05am UTC, it landed $45 \mathrm{~km}$ further north-east on a meadow next to Bad Salzungen (Thuringia).

\subsection{Telemetry}

Besides GPS, the payload was also equipped with several other sensors measuring the environment inside the styrofoam box. Figure 3 shows the recorded sensor data during the ascent. The temperature started at $30^{\circ} \mathrm{C}$ on the ground and gradually decreased to $-9^{\circ} \mathrm{C}$. The outside temperature at these altitudes is typically between $-50^{\circ} \mathrm{C}$ to $-60^{\circ} \mathrm{C}$, which demonstrates the

\footnotetext{
${ }^{2}$ http: //www. skysquitter.com

${ }^{3}$ https://www.rtl-sdr.com/buy-rtl-sdr-dvb-t-dongles/
} 


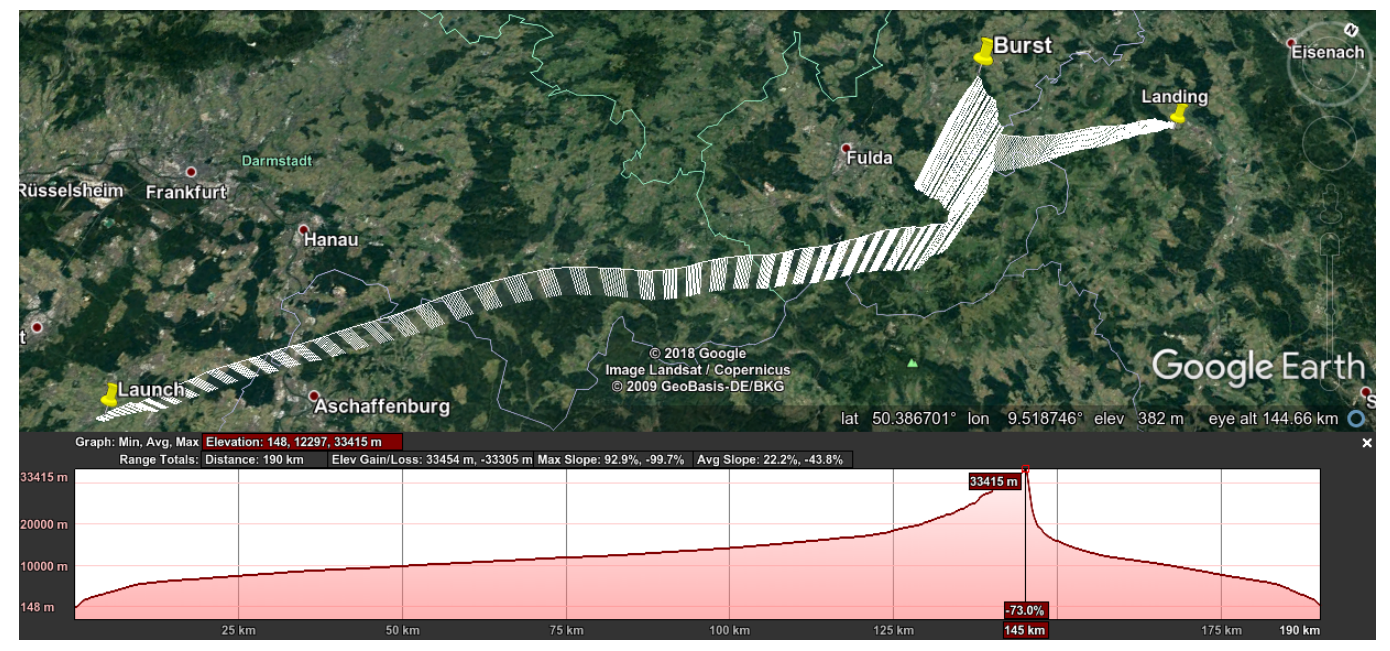

Figure 2: Flight path of the radio probe. It achieved a maximum altitude of $33.5 \mathrm{~km}$ and travelled more than $190 \mathrm{~km}$. Note that the map is slightly rotated and tilted for a better perspective.

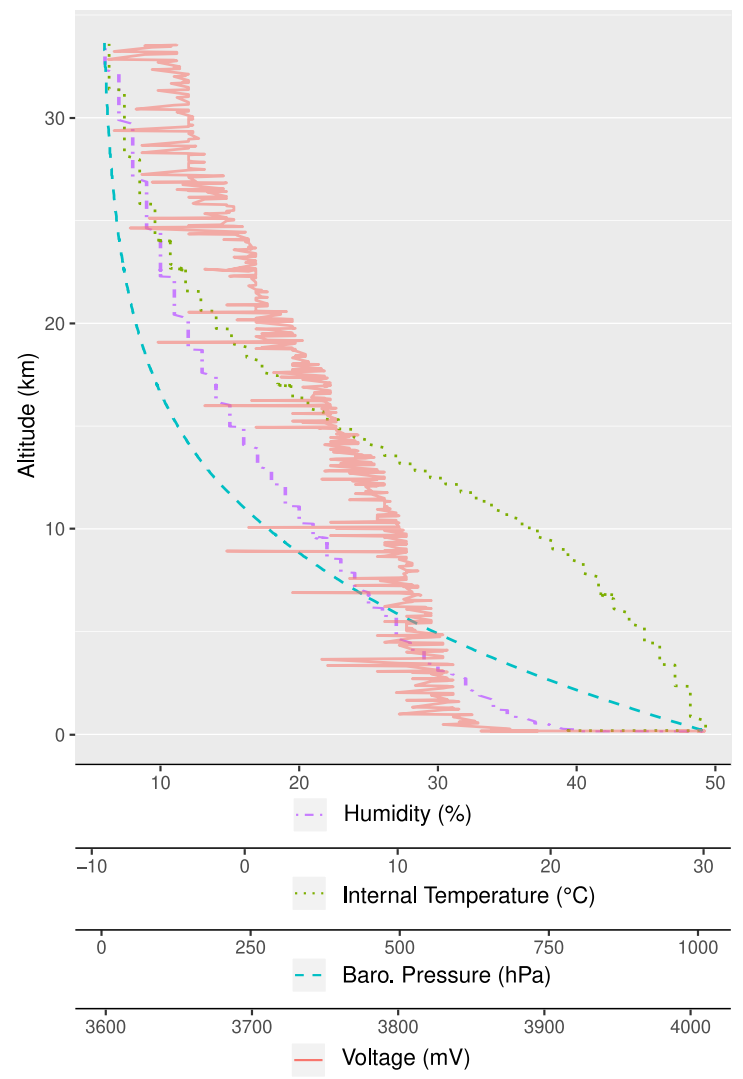

Figure 3: Measurements recorded by the onboard sensors during the ascent. The sensors were all located inside the styrofoam housing. 
insulation effect of the enclosure. At the same, the humidity inside the enclosure went from $49 \%$ down to $6 \%$ and the barometric pressure from $1007 \mathrm{hPa}$ down to $7 \mathrm{hPa}$. Since the box had no special sealing, this can be assumed to be equal to the outside pressure.

xThe voltage shown in Figure 3 can be used as an indicator for the charge left in the battery powering the payload. While the initial voltage (without load) was about $4 \mathrm{~V}$, it dropped to about $3.9 \mathrm{~V}$ once the payload was turned on and then gradually decreased to about $3.65 \mathrm{~V}$ when the burst altitude was reached. The sudden drops in voltage occur whenever the payload consumes more power, that is, whenever the Raspberry Pi and RTL-SDR are sampling signal data.

\section{Data Analysis}

In this section, we present our analysis of the data collected during the HAB's ascent. Our first focus will be on learning the characteristics and potential limitations of our experimental setup. This step is important to prevent drawing wrong conclusions and to improve the setup for future missions. In a second step, we will consider the $1090 \mathrm{MHz}$ frequency and its occupancy. The specific hypothesis that will be tested is whether the increasing altitude of the receiver and the resulting extension of its radio horizon leads to an increase of the experienced noise level since the number of transmitters (aircraft) within the HAB's line of sight also increases. Finally, we will analyze the ADS-B reception performance of the setup in terms of ranges and reception rates at higher altitudes.

\subsection{Data Preparation}

In order to analyze the data as described above, several processing steps are necessary to extract all required information from the raw I/Q data. First, we processed all $30 \mathrm{~s} \mathrm{I/Q}$ recordings and extracted physical metrics such as frequency occupancy, noise floor and average power. In addition, we calculated the spectrogram from the I/Q sample recordings to gain insights over the observed frequency band. Each spectrogram was averaged over an interval of $30 \mathrm{~s}$.

The second step was to extract the SSR Mode S and ADS-B data from the raw I/Q samples. To demodulate Mode S, we used a modified version of the latest dump $1090^{4}$ Mode S decoder. We modified the decoder such that it additionally estimates the noise floor for each decoded frame and outputs pseudo timestamps from offline I/Q recordings and other physical-layer metadata in CSV format. The noise floor was estimated based on the 500 samples preceding the detection. More specifically, we used the average signal strength (in dBFS) of those 125 out of these 500 samples with the lowest magnitude.

After demodulating the Mode S signals, we decoded the data produced by dump1090 using OpenSky's Java Mode S and ADS-B decoding library libadsb ${ }^{5}$. For every received Mode S transmission, we stored the unique transponder address of the transmitter and the downlink format. In the case of ADS-B position reports, we additionally stored the aircraft's location.

Finally, we enriched the decoded data with the balloon's telemetry data based on their timestamps. The balloon's exact position within the $30 \mathrm{~s}$ recordings was estimated by interpolating the positions reported in between. In the case where the aircraft position was also available, we additionally calculated its haversine distance and slant range to the balloon. This eventually allows us to analyse signal propagation characteristics and the high-altitude receiver's range.

\footnotetext{
${ }^{4}$ https://github.com/flightaware/dump1090

${ }^{5}$ https://github.com/openskynetwork/java-adsb
} 
It is worth noting that we only focused on SSR Mode S-based communications, i.e., SSR Mode S, ACAS, and ADS-B here. The main reason for this is that the other signals (mainly Mode A/C; see also subsection 2.1) contain only little information for a passive receiver and there are no open-source decoders available. However, their presence is still included in our frequency occupancy measurements.

\subsection{Results}

After applying the data preparation described in the previous section, we obtain data from three layers of the $1090 \mathrm{MHz}$ communication: physical layer data (I/Q samples, frequency occupancy, spectrogram, signal strength, noise floor), link layer data (timestamps and reception rates), and application layer data (transponder IDs and aircraft positions). In addition, we can join each of these subsets with the balloon's telemetry to correlate them with the probe's location and other sensor data at the time of reception.

\subsubsection{Measured Signal Strengths}

We start our analysis by looking at the measured signal strengths and how they correlate with the well-known free-space path loss model. Our goal is to validate the measurements and detect potential issues with the setup by comparing the data to a theoretical propagation model. The result of this correlation is shown in Figure 4a. While the dependency on the distance between transmitter and receiver is as expected, we also found a surprising dependency on the temperature inside the HAB's payload.

To further analyze whether the dependency on temperature is a side effect of some change in propagation characteristics due to the high altitude, we also compared the measured noise floor to the temperature in Figure 4b. If the increase in measured signal strengths was indeed caused by a change in propagation characteristics, the noise floor should not be affected in the same way. Figure $4 \mathrm{~b}$, however, shows that the magnitude of the noise floor also increased with decreasing temperature. The fact that both the signal strength and the noise floor increased suggests that the increase in measured signal strength is rather caused by changing characteristics of the measurement equipment caused by the changing environment.

Figure 4c additionally strengthens this theory as we could not observe any dependency of the signal-to-noise ratio (SNR) on the temperature. We conclude that with decreasing temperature the noise level increased exactly by the same factor as the measured signal strengths. This again indicates a change in the equipment's measurement characteristics, rather than a change in signal propagation characteristics or the radio environment. In fact, this negative dependency between measured signal strengths and environment temperature is a known effect [5]. Potential causes include the reduction of resistance and self-inductance due to skin effects and thermal contraction of wires.

\subsubsection{Spectrum Characteristics}

Figure $4 \mathrm{c}$ further indicates that the hypothesis mentioned in the introduction to this section is not true or at least not measurable with this setup. If it were, the SNR would become lower at higher altitudes (and thus at lower temperatures) as the noise level increases. There is, however, no visible correlation between the SNR and the probe's altitude. This is also shown in the spectrogram outlined in Figure 5. Given the sampling frequency of $2.4 \mathrm{MHz}$, the spectrum observed by the probe ranges from about $1088.8 \mathrm{MHz}$ to $1091.2 \mathrm{MHz}$. Yet, except for the general increase in power that can be attributed to the aforementioned temperature effect, there is no significant change in the spectrum visible at higher altitudes. Note that the 


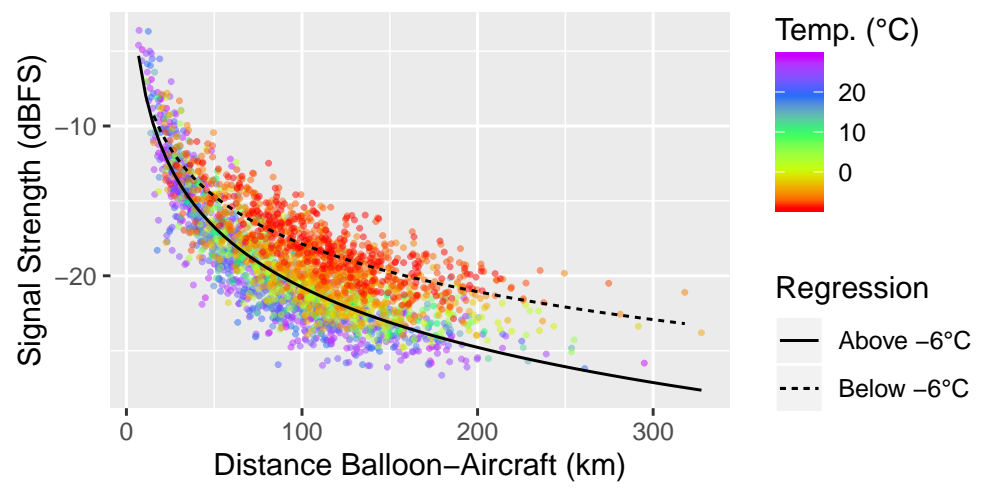

(a) The effect of distance and temperature on the received signal strength.

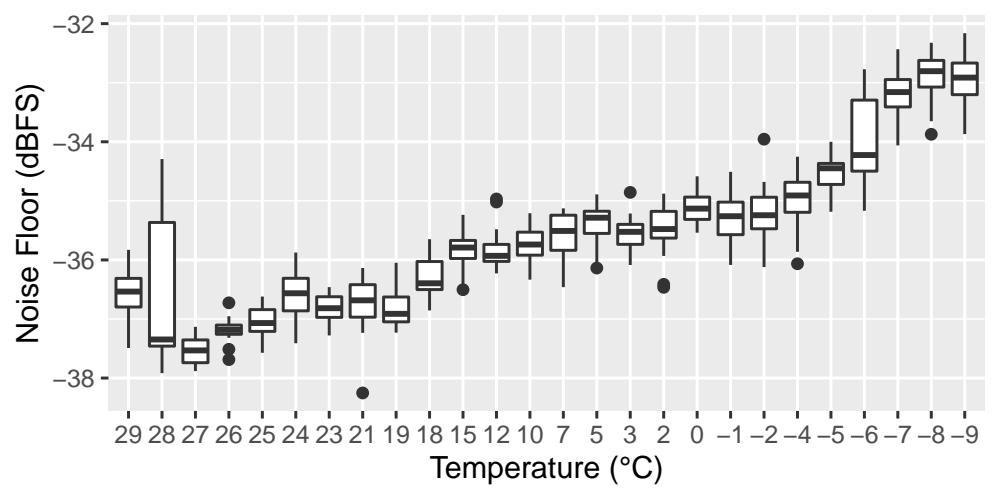

(b) The effect of temperature on the noise level observed during the Mode S reception.

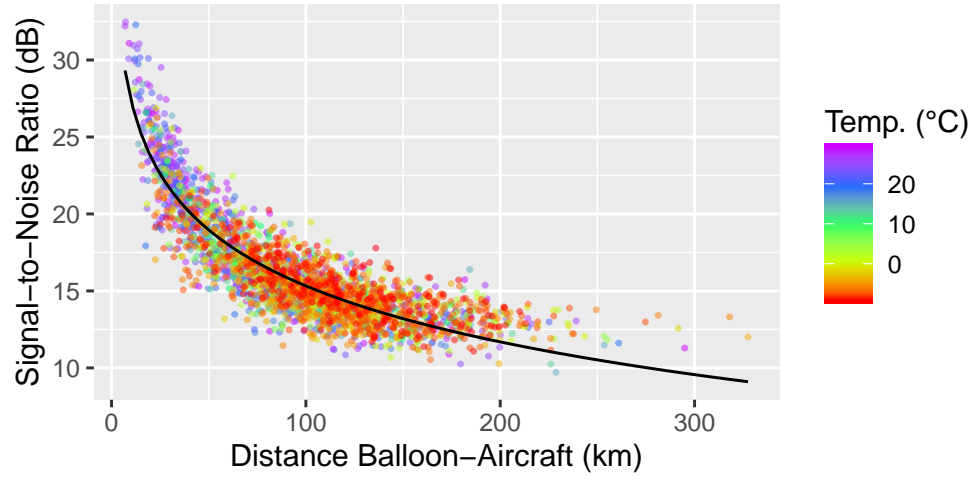

(c) Signal-to-noise ratio over distance and temperature.

Figure 4: The behavior of power measurements during the ascent. As expected, the received signal strength depends on the distance between transmitter and receiver. In addition, we could find an increasing offset of overall power measurements (i.e., in signals and noise) as the temperature decreased. 


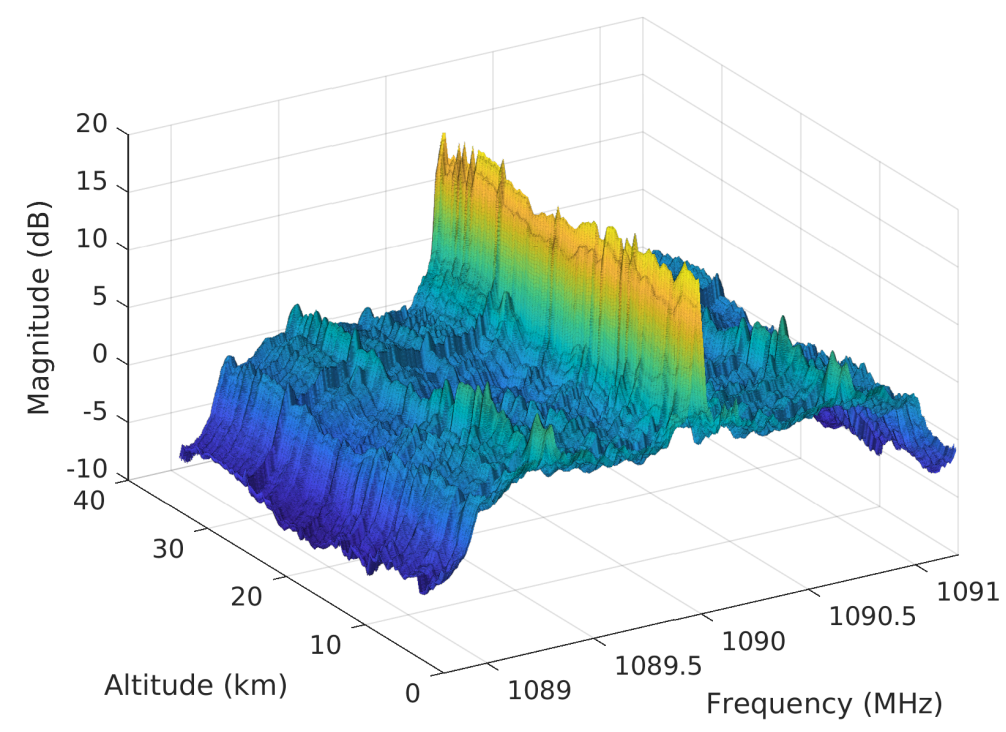

Figure 5: Spectrum observed during the ascent.

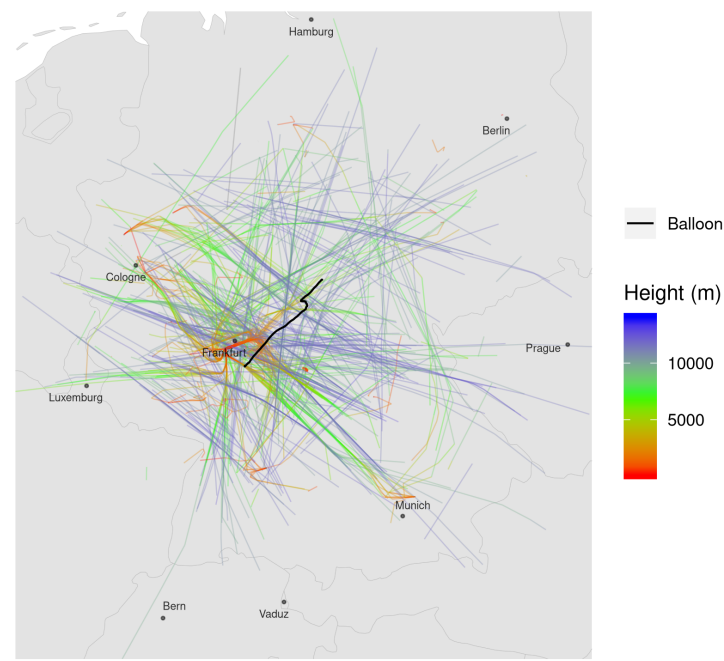

Figure 6: All flights observed via ADS-B during the ascent.

small peaks to the left and right from the center frequency are likely caused by harmonics and aircraft transmitting with a carrier frequency offset.

\subsubsection{Mode S \& ADS-B}

In this last part of the data analysis, we look at the Mode $\mathrm{S}$ and ADS-B data extracted from the raw I/Q samples. These data should give us a better impression of the overall reception 


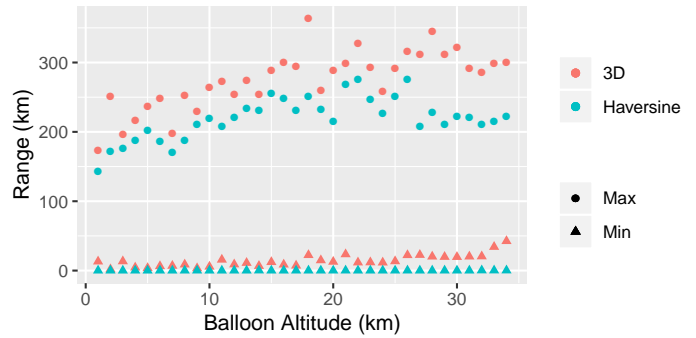

(a) Reception range during the ascent. The range was estimated based on the positions reported by aircraft via ADS-B and the probe's GPS sensor.

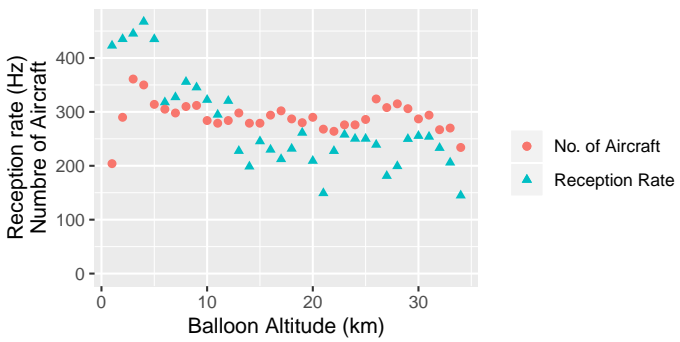

(b) Number of different transponder addresses seen at different altitudes and the average Mode S signal reception rate.

Figure 7: Reception range/messages rate analysis versus balloon altitude

performance of the setup during the ascent. An overview over all decoded ADS-B positions is provided in Figure 6. Major airports in vicinity such as Frankfurt am Main Airport, Munich Airport, and Cologne Bonn Airport are clearly visible due to the lower altitude traffic in their terminal manoeuvring areas. Overall, the probe picked up signals from all over Germany, with a clear dominance around Frankfurt, the most crowded airspace in this region.

The reception of $1090 \mathrm{MHz}$ signals is usually limited to line of sight communications. As many aircraft transmit signals with peak powers of 51-57 dBm, ranges of up to $1000 \mathrm{~km}$ (path loss of about $153 \mathrm{~dB}$ ) are possible, depending on the sensitivity of the receiver. Under the assumptions that there are no special geographical conditions such as mountains, a groundbased receiver can receive transponder signals from aircraft as far away as 400-450 km, given that en route aircraft are flying at altitudes of at least $10-15 \mathrm{~km}$. This theoretical radio horizon is further extended if the receiver's altitude increases. At a receiver altitude of $5 \mathrm{~km}$, the horizon is at about $700 \mathrm{~km}$, at an altitude of $20 \mathrm{~km}$, the horizon extends to about $1000 \mathrm{~km}$. A logical conclusion is the probe should receive signals from more and more aircraft during its ascent as its radio horizon and therefore its coverage expands.

Figure 7a shows the ranges calculated from the probe's telemetry and the received ADS-B signals. Initially, the maximum range was around $200-250 \mathrm{~km}$ and increased to about $300-$ $350 \mathrm{~km}$ at an altitude of $20 \mathrm{~km}$, yet, it stays well below the probe's theoretical radio horizon. There are three reasons why the probe's range does not expand with the radio horizon. First, the probe's sensitivity does not support reception of signals below a certain threshold. Second, an increasing noise level results in a lower SNR and therefore in a decrease of range. Third, collisions do not allow to receive remote airplanes. The analysis in subsubsection 4.2.1 (especially Figure 4c) indicates that the second reason is not the case.

It is worth mentioning that the RTL-SDR's sample rate is close to the Nyquist frequency of $2 \mathrm{MHz}$, the lower limit for receiving Mode $\mathrm{S}$ signals. In addition, the sample resolution of 8 bit is also rather low. Both things combined generally result in a rather small dynamic range, bad degarbling performance, a lower noise resistance, and a low sensitivity. In comparison, commercial SDR-based Mode S receivers have sample frequencies well beyond $10 \mathrm{MHz}$ and common sample resolutions of 12-16 bit. This leads us to the conclusion that the sensitivity of the probe was not sufficient to receive signals over distances of more than $350 \mathrm{~km}$.

This hypothesis is backed by the fact that the range never exceeded $350 \mathrm{~km}$, a rather bad performance when compared, e.g., to OpenSky's ground-based receivers which receive transmissions up to $500 \mathrm{~km}$. This might be a result of the low resolution and potentially high system 


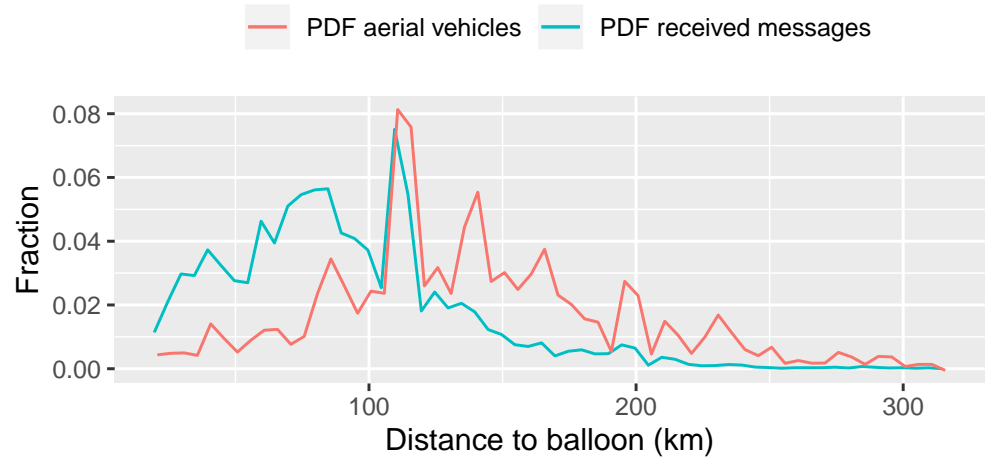

Figure 8: Comparison between received messages and visible aerial vehicles above $28 \mathrm{~km}$. The high peak is Frankfurt Main Airport where many vehicles are broadcasting.

loss. Using appropriate analog filters and a more optimized antenna might improve the range.

It is also worth noting that well-deployed ground-based receivers in Central Europe typically see up to 200 aircraft at the same time, whereas the probe constantly received signals from about 300 aircraft (see Figure 7b). The reason for this increased number of aircraft was that, unlike ground-based receivers, the probe also covered lower altitudes. As shown in Figure 6, major airports were within its coverage, likely being the cause for the increased number of aircraft.

Comparing our results with the simulated results in [12], we can observe that we measure significantly more devices than they predicted. Their simulation ignores a set of messages that do apply in this particular scenario. Collisions are therefore also a reason for the smaller range. This is backed by Figure 8, where the discrepancy between the received messages and the visible aerial vehicles is visualized. Clearly, significantly more messages are seen before the peek, representing Frankfurt Main Airport, than behind the peak. Therefore, remote vehicles have low probability of being received due to collisions, and this limits the range.

\section{Conclusion}

In this paper, we presented the results of our high-altitude radio frequency measurements. We used a high-altitude balloon equipped with a low-cost software-defined radio to collect raw I/Q signal data on the $1090 \mathrm{MHz}$ frequency at altitudes up to $33.5 \mathrm{~km}$. For our data analysis, we extracted and decoded Mode S and ADS-B signals from the raw I/Q samples. This allowed us to analyze the spectrum around $1090 \mathrm{MHz}$, the radio probe's reception performance, and signal propagation characteristics.

While HABs provide a low-cost platform to conduct such high-altitude measurement campaigns, several challenges must be considered. First, the onboard sensors must be able to operate under a wide temperature range. This is especially important for radio frequency measurement equipment which is susceptible to temperature-based effects. Therefore, temperature control and insulation are crucial to provide stable measurement performance. In the case of $1090 \mathrm{MHz}$, some temperature-induced effects such as amplified power measurements could also be removed offline by using signals from aircraft and the respective propagation models.

Second, the sensitivity and resolution of the onboard radio must meet the challenging radio environment at high altitudes. At higher altitudes, many more transmitters are in the range of the device whereas signals tend to be weak due to the long distances. Thus, a highly sensitive 
radio with a high resolution and low system loss might be required depending on the goal of the measurement campaign. This is especially challenging given the low power and low weight requirements of such a setup. Our experiment allows outlining the potential challenges air traffic surveillance through satellites might face in the future.

\section{Acknowledgements}

This research supported in part by armasuisse under the Cyber and Information Research Program, the NATO Science for Peace and Security Programme under grant G5461. We would like to thank the Team of Skysquitter for their support during and after the measurements and for providing their valuable insights during the data analysis and preparation of this paper.

\section{References}

[1] results from easa technical investigation on the radar detection losses in june 2014 in central europe. Technical report.

[2] Yuval Brodsky, Richard Rieber, and Tom Nordheim. Balloon-borne air traffic management (atm) as a precursor to space-based atm. Acta Astronautica, 70:112 - 121, 2012.

[3] N. Demidovich, P. Purcell, R. Dewey, T. Lachenmeier, C. Greenlow, T. Willson, J. DiNofrio, and D. Edwards. Dual frequency ads-b payload flight experiment on stratospheric balloon. In 2014 Integrated Communications, Navigation and Surveillance Conference (ICNS) Conference Proceedings, pages R1-1-R1-12, April 2014.

[4] R. Francis, R. Vincent, J. Noël, P. Tremblay, D. Desjardins, A. Cushley, and M. Wallace. The Flying Laboratory for the Observation of ADS-B Signals. International Journal of Navigation and Observation, 2011.

[5] T. Y. Kang and D. Seo. The effect of temperature on performance of a rf cmos power amplifer and bond wires. In 2015 IEEE 24th Electrical Performance of Electronic Packaging and Systems (EPEPS), pages 69-72, Oct 2015.

[6] Tim T Lachenmeier and Russ Dewey. Suitability of ads-b as a beacon for stratospheric balloon platforms and payloads. In AIAA Balloon Systems Conference, page 2912, 2015.

[7] M. Leonardi, S. Spinelli, and G. Galati. Ads-b/mlat surveillance system from high altitude platform systems. In 2011 Tyrrhenian International Workshop on Digital Communications - Enhanced Surveillance of Aircraft and Vehicles, pages 153-158, Sep. 2011.

[8] S. Nag, J. Rios, D. Gerhardt, and C. Pham. Cubesat constellation design for air traffic monitoring. Acta Astronautica, 128:180-193, 2016.

[9] S. Rajendran, R. Calvo-Palomino, M. Fuchs, B. Van den Bergh, H. Cordobés, D. Giustiniano, S. Pollin, and V. Lenders. ElectroSense: Open and big spectrum data. IEEE Communications Magazine, 56(1):210-217, 2018.

[10] M. Schafer, M. Strohmeier, M. Smith, M. Fuchs, R. Pinheiro, V. Lenders, and I. Martinovic. Opensky report 2016: Facts and figures on ssr mode s and ads-b usage. In 2016 IEEE/AIAA 35th Digital Avionics Systems Conference (DASC), pages 1-9, Sep. 2016.

[11] V. Schejbal, P. Bezousek, J. Pidanic, and M. Chyba. Secondary surveillance radar antenna. IEEE Antennas and Propagation Magazine, 55(2), April 2013.

[12] Richard Van Der Pryt and Ron Vincent. A simulation of signal collisions over the north atlantic for a spaceborne ads-b receiver using aloha protocol. Positioning, 6(03):23, 2015. 